\title{
REMARKS ON THE BOUNDARY ELEMENT METHOD FOR STRONGLY NONLINEAR PROBLEMS
}

\author{
KEIJO RUOTSALAINEN ${ }^{1}$
}

(Received 29 May 1990; revised 23 October 1991)

\begin{abstract}
Recently in several papers the boundary element method has been applied to nonlinear problems. In this paper we extend the analysis to strongly nonlinear boundary value problems. We shall prove the convergence and the stability of the Galerkin method in $L^{p}$-spaces. Optimal order error estimates in $L^{p}$ space then follow. We use the theory of $A$-proper mappings and monotone operators to prove convergence of the method. We note that the analysis includes the $u^{4}$-nonlinearity, which is encountered in heat radiation problems.
\end{abstract}

\section{Introduction}

For some time the analysis of boundary element methods (i.e. BEM) has interested many authors. At the same time the development of computational software has made the boundary element method an alternative to the more conventional finite element techniques in engineering applications. And when the implementation of the boundary element method is done ingeniously (cf. [13] for the panel clustering method), it will be in fact as efficient in computing the approximate solution to linear elliptic boundary value problems as other methods available. The efficiency here refers to both complexity and accuracy of the method.

The purpose of this paper is to extend the analysis of the boundary element methods to nonlinear problems started in [21]. There the Galerkin boundary element method was studied for the first time for a mildly nonlinear boundary integral equation, which was obtained by the direct formulation of the nonlinear boundary value problem. Later in [20] the analysis was extended

\footnotetext{
${ }^{1}$ University of Oulu, Faculty of Technology, Section of Mathematics, SF-90570 Oulu, Finland.

(C) Copyright Australian Mathematical Society 1993, Serial-fee code 0334-2700/93
} 
to the collocation method, which seems to be easier to implement than the Galerkin method (cf. [14]). In [22], which contains more general Hammerstein equations, the analysis has been sharpened to yield also $L^{2}$-estimates. Recently, optimal $L^{p}$-estimates, $1 \leq p \leq \infty$, were derived by Eggermont and Saranen [10]. However, all the works mentioned assume that the nonlinearity is mild, having at most linear growth.

Here we shall allow strong nonlinearities, which permits us to study the numerical approximation of the black-body radiation with the $u^{4}$-nonlinearity. In the papers mentioned above and here, we shall study the numerical approximation of the nonlinear boundary value problem

$$
\begin{cases}\Delta \Phi=0, & \text { in } \Omega, \\ -\partial_{n} \Phi=G(\Phi)-f, & \text { on } \Gamma .\end{cases}
$$

Here $\Gamma$ is a smooth simple closed curve and $\Omega$ is the bounded region enclosed by $\Gamma$. The symbol $\partial_{n}$ is already standard for the outer normal derivative on $\Gamma$. Note that the differential operator is linear, the nonlinearities appearing only on the boundary. This is not a severe restriction. Via the Kirchhoff transformation, more general quasi-linear equations can be brought into this form.

Here we shall consider the indirect formulation of the boundary value problem (1). We shall make the ansatz: Find a boundary distribution $u$ (in some appropriate function space defined later on) such that

$$
\Phi(x)=-\frac{1}{2 \pi} \int_{\Gamma} u(y) \log |x-y| d s_{y}, \quad x \in \Omega .
$$

Then by the properties of the normal derivative of the monopole potential $[26,6,7]$ we derive (see Section 2 ) the nonlinear boundary integral equation

$$
\left(\frac{1}{2} I-K^{*}\right) u+G(V u)=f
$$

Here the operator $K^{*}$ is the spatial adjoint of the double layer operator $K$, which is defined by

$$
K u(x)=\frac{1}{2 \pi} \int_{\Gamma} u(y) \partial_{n} \log |x-y| d s_{y}
$$

and the single layer operator $V$ is defined by

$$
V(u)(x)=-\frac{1}{2 \pi} \int_{\Gamma} u(y) \log |x-y| d s_{y}, \quad x \in \Gamma .
$$

In the next section we collect the basic mapping properties of operators in question. There the necessary function spaces are defined and the fundamental properties are recalled. Especially we introduce the exact form of the 
nonlinearities which are treated throughout the paper. We impose the necessary growth conditions that will fix the function spaces in which the boundary integral equations are studied, and collect all the assumptions that the nonlinearity should fulfil in order to yield a unique solution to the nonlinear boundary integral equation (3).

The existence and uniqueness are then discussed in the third section. Using the variational methods in the standard manner, the existence of the solution is established. Since the integral operator defined by the formula (3) is strictly $V^{-1}$-monotone, we finally obtain the uniqueness of the solution. To ensure this to be true, we assume that the nonlinearity is a strictly monotone real function. These properties are crucial when we discuss the uniqueness of the approximate solution.

After studying the mapping properties of the continuous problem, we then focus attention on the study of the approximate scheme. In the fourth section we introduce the necessary approximation spaces and the necessary approximation properties they possess. After that we show that the Galerkin equations are uniquely solvable. In this context we use the theory of $A$ proper mappings and the degree theory of nonlinear mappings to obtain the existence of the approximate solution and the convergence of the method considered. Finally we shall study the the asymptotic error estimates of the Galerkin method in $L^{p}$-spaces. The compact operators in (3) are in fact pseudodifferential operators of order -1 . When these operators are applied to some $L^{p}$-spaces, the images are more regular. Utilising this property we may derive error estimates in weaker norms than the $L^{p}$-topology. This provides better estimates for the approximate potential of (1).

\section{The mapping properties}

Let $\Omega$ be a bounded open domain in the plane $\mathbf{R}^{2}$ with a smooth boundary $\Gamma=\partial \Omega$. In other words $\Gamma$ has a regular parameter representation $x: \mathbf{R} \rightarrow \Gamma$ with nonvanishing Jacobian, i.e. $\left|\frac{d x}{d t}\right| \neq 0$ and the representation is a $C^{\infty}$ mapping. To avoid difficulties related to the mapping properties of the single layer operator (i.e. the Symm's operator) we assume that the conformal capacity $\operatorname{cap}(\Gamma) \neq 1$.

Let us now examine more closely the class of problems that are to be treated: find $\Phi$ such that

$$
\begin{cases}\Delta \Phi=0, & \text { in } \Omega, \\ -\partial_{n} \Phi=G(\Phi)-f, & \text { on } \Gamma,\end{cases}
$$

where $f$ and $G(\cdot)$ are functions with various properties which we shall specify next. 
The nonlinear mapping $G$ is the Nemitskyi operator corresponding to the Carathéodory function $g(\cdot, \cdot): \Gamma \times \mathbf{R} \rightarrow \mathbf{R}$. We remind the reader that a function $g(\cdot, \cdot)$ fulfils the Carathéodory conditions whenever

A1. $g(\cdot, u): \Gamma \rightarrow \mathbf{R}$ is measurable for all $u \in \mathbf{R}$;

A2. $g(x, \cdot): \mathbf{R} \rightarrow \mathbf{R}$ is continuous for almost all $x \in \Gamma$.

In the sequel, we shall follow the convention that whenever $g(\cdot)$ is used it is meant to represent the function $g(x, \cdot)$ for almost every $x \in \Gamma$. In contrast to $[10,19,20,21,22]$ we do not require $g(\cdot)$ to have a linear growth. Instead, we shall assume that it possesses the following properties for some fixed $p \geq 2$ :

A3. there exist constants $a_{1}>0, a_{2} \geq 0$ independent of $u$ such that

$$
|g(u)| \leq a_{1}|u|^{p-1}+a_{2}, \quad g(u) u \geq b_{1}|u|^{p}+b_{2} ;
$$

A4. $g(\cdot)$ is strictly increasing function on $\mathbf{R}$;

A5. there exist constants $K_{1}>0$ and $K_{2} \geq 0$ such that

$$
|g(u)-g(v)| \leq|u-v|\left\{K_{1}+K_{2}\left(|u|^{p-2}+|v|^{p-2}\right)\right\} .
$$

Before proceeding to reformulate the boundary value problem as a boundary integral equation, we first introduce some notations. By $L^{p}(\Gamma)$ we denote the conventional Banach space with respect to the Lebesgue measure on $\Gamma$. This space is endowed with the usual $L^{p}$-norm, $1 \leq p<\infty$,

$$
\|u\|_{L^{p}(\Gamma)}=\left\{\int_{\Gamma}|u(x)|^{p} d s_{x}\right\}^{1 / p}
$$

We remind the reader that these spaces are reflexive. The dual spaces are defined with respect to the $L^{2}$-inner product. It is an already well-known fact that the dual space of $L^{p}(\Gamma)$ is $L^{q}(\Gamma)$, where $q$ is the conjugate exponent: $\frac{1}{p}+\frac{1}{q}=1$.

To distinguish the function spaces defined on the domain $\Omega$ from the one on the boundary, the $L^{p}$-spaces in $\Omega$ will be denoted by $L^{p}(\Omega)$ and the corresponding norm will then be $\|\cdot\|_{p, \Omega}$. In the course of the paper, the Sobolev spaces are also needed. On the boundary $W^{t, p}(\Gamma)$ stands for the conventional Sobolev-Slobodetskii space for $t>0$. The negative-order Sobolev spaces are then defined by duality with respect to the $L^{2}$-inner product. Other related function spaces considered in this paper will be defined in the order of occurence when necessary.

We start our consideration by recalling some basic mapping properties of the single and double layer operators (cf. [10]): 
THEOREM 1. The boundary operators $V, K, K^{*}$ have the following mapping properties:

(1) $V, K, K^{*}: L^{p}(\Gamma) \rightarrow L^{q}(\Gamma)$ are bounded and compact for all $p, q \in[1, \infty]$.

(2) $V, K, K^{*}: L^{p}(\Gamma) \rightarrow C(\Gamma)$ are compact.

(3) $V: W^{t, 2}(\Gamma) \rightarrow W^{t+1,2}(\Gamma)$ is an isomorphism for all $t \in \mathbf{R}$.

Next we shall establish the mapping properties of the Nemitskyi operator $G(\cdot): L^{p}(\Gamma) \rightarrow L^{q}(\Gamma)$ corresponding to a Carathéodory function satisfying the assumptions A1-A5 above.

LEMMA 2. The Nemitskyi operator $G: L^{p}(\Gamma) \rightarrow L^{q}(\Gamma)$ is bounded, continuous and strictly monotone, i.e. for all $u, v \in L^{p}(\Gamma)$ there holds

$$
(G(u)-G(v), u-v)_{L^{2}(\Gamma)} \geq 0 .
$$

The equality in (7) holds only if $u=v$.

Proof. The statement is now a direct consequence of the well-known properties of the Nemitskyi operators. The proof can be found in any text book [17, Chapter IV, Section 1].

Finally we have to impose one more restrictive condition on the nonlinearity. Namely, we suppose that the partial derivative satisfies the following growth condition:

$$
\left|\frac{\partial}{\partial u} g(u)\right| \leq b+a|u|^{p-2},
$$

where $a$ and $b$ are some nonnegative constants. Then the Nemitskyi operator is also Fréchet-differentiable from $L^{p}(\Gamma) \rightarrow L^{q}(\Gamma)$ and the derivative is given by

$$
D G(u) v(x)=\frac{\partial}{\partial u} g(x, u(x)) v(x)
$$

for almost all $x \in \Gamma$.

In this occasion we note that the Carathéodory function

$$
g(x, u)=|u| u^{3}
$$

satisfies all the assumptions A1-A5 and the additional growth condition (8). But it has even stronger properties. Its derivative function $\frac{\partial}{\partial u} g(u)=$ $4|u|^{3}$ is also a Carathéodory function. Since the related Nemitskyi operator is bounded and continuous from $L^{5}(\Gamma) \rightarrow L^{\frac{5}{3}}(\Gamma)$, then by [25, Chapter 20] the Nemitskyi operator $G: L^{5}(\Gamma) \rightarrow L^{\frac{5}{4}}(\Gamma)$ is continuously Fréchetdifferentiable. 
Motivated by the previous considerations we make the following assumption, which we will use in the forthcoming sections. Besides the conditions A1-A5 above we require:

A6. The Nemitskyi operator corresponding to the Carathéodory function

$$
\frac{\partial}{\partial u} g(x, u): \Gamma \times \mathbf{R} \rightarrow \mathbf{R}
$$

is bounded and continuous from $L^{p}(\Gamma)$ to $L^{\frac{p}{p-2}}(\Gamma)$.

Under this additional assumption the following lemma is valid [25, Chapter 20]:

LemMa 3. The Nemitskyi operator $G: L^{p}(\Gamma) \rightarrow L^{q}(\Gamma)$ is continuously Fréchetdifferentiable.

Combining the results of Theorem 1 and Lemmas 2 and 3, one easily verifies the following statement:

Theorem 4. Let us assume that the Caratheodory function $g(\cdot): \Gamma \times \mathbf{R} \rightarrow \mathbf{R}$ fulfills the assumptions A1-A6. Then the nonlinear operator

$$
A(u)=\left(\frac{1}{2} I-K^{*}\right) u+G(V u)
$$

is bounded, continuously Fréchet-differentiable in $L^{q}(\Gamma)$.

Proof. For every $u \in L^{q}(\Gamma)$ we have by the triangle inequality

$$
\|A(u)\| \leq \frac{1}{2}\|u\|_{L^{q}(\Gamma)}+\left\|K^{*} u\right\|_{L^{q}(\Gamma)}+\|G(V u)\|_{L^{q}(\Gamma)} .
$$

Using the growth condition $\mathbf{A 3}$ and the Minkowski inequality we obtain

$$
\begin{aligned}
{\left[\int_{\Gamma}|G(V u)(x)|^{q} d s_{x}\right]^{1 / q} } & \leq\left[\int_{\Gamma} \alpha_{1}^{q}|V u(x)|^{(p-1) q} d s_{x}\right]^{1 / q}+m(\Gamma) \alpha_{2} \\
& =\alpha_{1}\|V u\|_{L^{p}(\Gamma)}^{p / q}+m(\Gamma) \alpha_{2} .
\end{aligned}
$$

Here we used the property $(p-1) q=p$ for the conjugate exponents $p$ and $q$. Now the boundedness follows from Theorem 1 , which states that

$$
\begin{aligned}
\left\|K^{*} u\right\|_{L^{q}(\Gamma)} & \leq c\|u\|_{L^{q}(\Gamma)}, \\
\|V u\|_{L^{p}(\Gamma)} & \leq c\|u\|_{L^{q}(\Gamma)} .
\end{aligned}
$$

The continuity of the mapping is clear from the mapping properties of $V, K^{*}$ and $G$. It remains to prove the Fréchet-differentiability. By the definition of 
the Fréchet-derivative it is sufficient to show that the linear operator $D A(u)$ defined by setting

$$
D A(u) h=\left(\frac{1}{2} I-K^{*}\right) h+D G(V u) V h
$$

is bounded as an operator from $L^{q}(\Gamma)$ into itself, and that

$$
\lim _{\|h\|_{L^{q}(\Gamma)} \rightarrow 0} \frac{\|A(u+h)-A(u)-D A(u) h\|_{L^{q}(\Gamma)}}{\|h\|_{L^{q}(\Gamma)}}=0 .
$$

The definition of the mapping $A(\cdot)$ yields immediately that

$$
A(u+h)-A(u)=\left(\frac{1}{2} I-K^{*}\right) h+G(V(u+h))-G(V u) .
$$

Therefore we have by the formula (14)

$$
A(u+h)-A(u)-D A(u) h=G(V(u+h))-G(V u)-D G(V u) V h
$$

and by Lemma 3 there holds the estimate

$$
\begin{aligned}
& \|G(V(u+h))-G(V u)-D G(V u) V h\|_{L^{q}(\Gamma)} \\
& \quad=\|V h\|_{L^{p}(\Gamma)} R\left(\|V h\|_{L^{p}(\Gamma)}\right) \leq c\|h\|_{L^{q}(\Gamma)} R\left(\|h\|_{L^{q}(\Gamma)}\right)
\end{aligned}
$$

where $R(r) \rightarrow 0$, as $r \rightarrow 0^{+}$. But this estimate already proves the Fréchetdifferentiability. The continuity of the Fréchet-derivative is obvious, since $u \rightarrow D G(V u)$ is continuous by Lemma 3. This completes the proof of the statement.

\section{Existence and uniqueness}

The standard approach to deal with the existence and uniqueness of the solution for a nonlinear equation requires usually that a nonlinear operator is monotone, coercive, bounded and continuous (cf. Theorem 27.1 in [16]). In our case we shall use some generalisation of the concept of a monotone operator. Since the monotonicity in the existence and uniqueness proofs is usually used only in the last step, when one proves the uniqueness of a solution, we can find in the literature a multitude of useful generalised concepts.

The nonlinear Hammerstein operator $A(u)$ is not monotone, but if "we change the coordinates" in some sense, they possess a related property. Namely, by using the properties of the single and double layer operators we are able to show 
THEOREM 5. The Hammerstein operator $A(\cdot): L^{q}(\Gamma) \rightarrow L^{q}(\Gamma)$ is a strictly $V$-monotone mapping, i.e. for all $u, v \in L^{q}(\Gamma), u \neq v$ there holds

$$
(A(u)-A(v), V(u-v))_{L^{2}(\Gamma)}>0 .
$$

Proof. To establish the monotonicity property we shall quote the following mapping property [21, Theorem 2, p. 303]: For every $\chi \in H^{\frac{1}{2}}(\Gamma)=W^{\frac{1}{2}, 2}(\Gamma)$ there holds

$$
\left(V^{-1}\left(\frac{1}{2} I-K\right) \chi, \chi\right)_{L^{2}(\Gamma)}=\int_{\Omega}|\nabla \Psi|^{2} d x
$$

where $\Psi \in W^{1,2}(\Omega)$ is the harmonic function having $\chi$ as its boundary value, i.e. $\left.\Psi\right|_{\Gamma}=\chi$, and $V^{-1}$ is the inverse of the single layer operator.

Let us assume that $u, w \in L^{q}(\Gamma)$. Then by Theorem 1 the function $V(u-w)$ is contained in the Sobolev space $W^{1, q}(\Gamma)$. Since we assumed that

$$
\rho=1-\left(\frac{1}{q}-\frac{1}{2}\right)>0,
$$

we have by Theorem 7.58 in [1] the continuous imbedding

$$
W^{1, q}(\Gamma) \hookrightarrow W^{\frac{1}{2}, 2}(\Gamma) \text {. }
$$

Therefore $V(u-w)$ is also contained in the Sobolev-Slobodetskii space $W^{\frac{1}{2}, 2}(\Gamma)$. Setting in (20) $\chi=V(u-w)$ we get, remembering that $V^{-1}$ is formally symmetric,

$$
\begin{aligned}
\int_{\Omega}|\nabla \Psi|^{2} d x & =\left(V^{-1}\left(\frac{1}{2} I-K\right) V(u-w), V(u-w)\right)_{L^{2}(\Gamma)} \\
& =\left(V(u-w),\left(\frac{1}{2} I-K^{*}\right)(u-w)\right)_{L^{2}(\Gamma)} .
\end{aligned}
$$

Using (21) we obtain finally the desired result

$$
\begin{aligned}
& (A(u)-A(w), V(u-w))_{L^{2}(\Gamma)}=\int_{\Omega}|\nabla \Psi|^{2} d x \\
& \quad+\int_{\Omega}[g(x, V u(x))-g(x, V w(x))][V u(x)-V w(x)] d x .
\end{aligned}
$$

Here the right-hand side is strictly positive for every $u \neq w$, since by the assumption $\mathbf{A 4}$ the function $g(u)$ is a strictly increasing function on $\mathbf{R}$.

This theorem yields us as a consequence the following.

COROLlaRY 6. The solution $u \in L^{q}(\Gamma)$ of the nonlinear integral equation

$$
A(u)=f
$$

is unique for every $f \in L^{q}(\Gamma)$. 
Next we shall proceed with the solvability of the boundary integral equations. We turn our attention to the equivalent boundary value problem:

Problem 1. Find $\Psi \in W^{1,2}(\Omega)$ such that

$$
\Delta \Psi=0, \quad \partial_{n} \Psi=G(\Psi)-f .
$$

It is well known that this potential problem is equivalent to the minimisation of the functional

$$
\mathscr{J}(\Psi)=\frac{1}{2} \int_{\Omega}|\nabla \Psi|^{2} d x+F(\Psi)-\int_{\Gamma} f g d s_{\Gamma}
$$

over $W^{1,2}(\Omega)[4,5,16]$. The functional $F(\cdot)$ depends on the boundary values of the function $\Psi \in W^{1,2}(\Omega)$. It is defined by setting

$$
F(\Psi)= \begin{cases}\int_{\Gamma} j(\Psi) d s_{x}, & j(\Psi) \in L^{1}(\Gamma), \\ +\infty, & \text { otherwise. }\end{cases}
$$

The function $j(\cdot)$ is strictly convex and lower semicontinuous and the subdifferential (subgradient) is given by

$$
\partial j(u)=G(u) .
$$

The existence of this function is verified in [4, Theorem 2.3], because $g(u)$ is a strictly monotone, proper function. For the function $j(\cdot)$ we have the explicit expression

$$
j(\Psi(x))=\int_{0}^{\Psi(x)} g(x, s) d s .
$$

By the growth condition $\mathbf{A 3}$, for every $\Psi \in W^{1,2}(\Omega)$ there exist constants $c>0, M \in \mathbf{R}$ independent of $\Psi$ such that

$$
F\left(\left.\Psi\right|_{\Gamma}\right) \geq c \int_{\Gamma}|u(x)|^{p} d s_{x}+M \int_{\Gamma}|u(x)| d s_{x},
$$

where $u=\left.\Psi\right|_{\Gamma}$. Note that according to the Sobolev imbedding theorem [1, Theorem 7.58] the Sobolev space $W^{1,2}(\Omega)$ is continuously imbedded in $L^{p}(\Gamma)$. In any case from this estimate it follows that the functional $\mathscr{J}$ is coercive:

$$
\begin{aligned}
\mathscr{J}(\Psi) & =\frac{1}{2} \int_{\Omega}|\nabla \Psi|^{2} d x+\int_{\Gamma} j(\Psi) d s_{x}-\int_{\Gamma} f \Psi d s_{x} \\
& \geq \frac{1}{2} \int_{\Omega}|\nabla \Psi|^{2} d x+c(p) \int_{\Gamma}|\Psi|^{p} d s_{x}-\|f\|_{L^{q}(\Gamma)}\|\Psi\|_{L^{p}(\Gamma)}
\end{aligned}
$$

Once the functional is also strictly convex and continuous, by the standard argument we can prove the existence of the function $\Psi \in W^{1,2}(\Omega)$, which is the minimiser of the functional $\mathscr{J}$ over the Sobolev space $W^{1,2}(\Omega)$. 
Now the minimiser of the functional in question is also the unique solution of the boundary value problem $(24)[5,16]$. Our problem is, however, to prove the existence of the solution to the nonlinear boundary integral equation (23).

THEOREM 7. For every $f \in L^{q}(\Gamma)$ there exists a unique solution $u \in L^{q}(\Gamma)$ of (23).

Proof. Let $\Psi \in W^{1,2}(\Omega)$ be the unique solution of the corresponding boundary value problem (24). By Theorem 1 there exists a uniquely determined boundary distribution $u \in W^{-\frac{1}{2}, 2}(\Gamma)$ such that $V(u)=\left.\Psi\right|_{\Gamma}$, and the function $\Psi$ has the integral representation

$$
\Psi(x)=S u(x)=-\frac{1}{2 \pi} \int_{\Gamma} u(y) \log |x-y| d s_{y} .
$$

Since the normal derivative of the single layer potential $S$ has the jump across the boundary [26], the normal derivative of $S(u)$ from the interior domain is given as

$$
\left.\partial_{n} \Psi\right|_{\Gamma}=\left[\frac{1}{2} I-K^{*}\right] u
$$

On the other hand by the boundary conditions in (24) there holds the relation

$$
-\left(\frac{1}{2} I-K^{*}\right) u=G(V u)-f
$$

in the topology of the Sobolev-Slobodetskii space $W^{-\frac{1}{2}, 2}(\Gamma)$. We can rewrite this equation in the form

$$
u=2 K^{*} u-2 G(V u)-2 f .
$$

From this form one immediately concludes that $u$ is contained in $L^{q}(\Gamma)$. Namely, by the mapping properties of $K^{*}$ and the single layer operator there holds (Theorem 1): $K^{*} u \in W^{\frac{1}{2}, 2}(\Gamma)$ and $V u \in W^{\frac{1}{2}, 2}(\Gamma)$. Using the Sobolev imbedding theorem which states that the continuous imbeddings

$$
W^{\frac{1}{2}, 2}(\Gamma) \hookrightarrow L^{p}(\Gamma), \quad 1<p<\infty,
$$

are valid, we obtain the inclusions

$$
K^{*} u \in L^{q}(\Gamma), \quad V u \in L^{p}(\Gamma) .
$$

Furthermore, $G$ maps $L^{p}(\Gamma)$ onto $L^{q}(\Gamma)$ continuously, implying the inclusion

$$
G(V u) \in L^{q}(\Gamma) .
$$

Combining these inclusions with (34) we obtain the $L^{q}$-regularity of the boundary distribution, since $f \in L^{q}(\Gamma)$ by the assumption. 
Thus we have proved the existence at least one solution. The uniqueness was already proved in Corollary 6 . The proof of the statement is now complete.

\section{The boundary element method}

Next we shall focus our attention on the numerical approximation of the nonlinear integral equation

$$
A(u)=\left(\frac{1}{2} I-K^{*}\right) u+G(V u)=f .
$$

We shall utilise the theory of $A$-proper mappings introduced and studied intensively by W. V. Petryshyn and F. Browder in the late 1960s. The concept is rather weak: it does not say anything about the existence of the finite dimensional approximate solutions. But combining this property with other mapping properties of the Hammerstein operator in question we achieve the unique solvability of the Galerkin equations.

Let us first fix the boundary element spaces used for the approximation. To this end we select a sequence of mesh points $\Theta=\left\{x_{i} \mid i=0, \ldots, N-1\right\}$ on $\Gamma$, and denote by $S_{N}^{d}(\Theta)$ the boundary element spaces transplanted from the space of 1-periodic, $(d-1)$-times continuously differentiable splines of degree $d$ onto $\Gamma$. In general, we assume the family of partitions to be quasiuniform, e.g. $\frac{\max \left(h_{i}\right)}{\min \left(h_{j}\right)} \in\left[\gamma, \gamma^{-1}\right]$ for all partitions in the family with some constant $\gamma>0$. Here we have used a natural notation $h_{i}=\left|x_{i+1}-x_{i}\right|$. The mesh parameter, however, is denoted by $h=1 / N$.

With the assumptions made above there holds the very well-known approximation and inverse properties $[2,3,11,24]$ :

Approximation property. For every $u \in W^{s, 2}(\Gamma), s \leq d+1$, there exists $\psi \in S_{N}^{d}(\theta)$ such that

$$
\|u-\psi\|_{W^{t, 2}(\Gamma)} \leq c h^{s-t}\|u\|_{W^{s, 2}(\Gamma)}
$$

where $t \leq s, t<d+\frac{1}{2}$. The constant depends only on $s, t$ and $d$; but not on the mesh parameter.

Inverse estimate. For all $\psi \in S_{N}^{d}(\Theta)$ there holds the inverse estimate

$$
\|\psi\|_{W^{s, 2}(\Gamma)} \leq c h^{t-s}\|\psi\|_{W^{t, 2}(\Gamma)},
$$

for $t \leq s<d+\frac{1}{2}$.

In this context we would like to remind the reader that the approximation property holds for general meshes. As a consequence for the $L^{2}$-projection 
the following estimates are valid [20]:

$$
\left\|\left(I-P_{h}\right) u\right\|_{W^{t, 2}(\Gamma)} \leq c h^{s-t}\|u\|_{W^{s, 2}(\Gamma)}
$$

for $-d-1 \leq t \leq s \leq d+1,-d-1 / 2<s$ and $t<d+1 / 2$.

But besides these conventional $L^{2}$-estimates we shall require the approximation properties also with respect to the $L^{p}$-topology. In the standard manner one then derives the uniform boundedness of the $L^{2}$-projection in $L^{p}$-spaces by using the Riesz-Thorin theorem [8] (see also [10, Lemma 6.2]). Furthermore, the $L^{2}$-projection of a function $u \in W^{s, p}(\Gamma), s \leq d+1$, possesses the error estimates

$$
\left\|u-P_{h} u\right\|_{W^{i, p}(\Gamma)} \leq c h^{s-t}\|u\|_{W^{s, p}(\Gamma)},
$$

where $-d-1 \leq t \leq \min \{s, 0\}$. Note that this result holds for entirely general meshes.

We shall approximate the solution of the nonlinear integral equation (23) by spline functions. In general, the problem is to find the coefficients $\alpha_{i} \in \mathbf{R}$, $i=0, \ldots, N-1$, such that

$$
u_{h}=\sum_{i=0}^{N-1} \alpha_{i} \psi_{i}
$$

is a good approximation of $\mathrm{u}$. The functions $\psi_{i}$ form a suitable basis of the spline space $S_{N}^{d}(\Theta)$.

We shall fix the coefficients by requiring that $u_{h}$ satisfies the Galerkin equations

$$
\left(A\left(u_{h}\right), \psi\right)_{L^{2}(\Gamma)}=(A(u), \psi)_{L^{2}(\Gamma)}
$$

for all $\psi \in S_{N}^{d}(\Theta)$. This choice leads us to solve a nonlinear system of equations.

Possibly there exist several conceptual frameworks to prove the existence of the approximate solution, and what is most important, the convergence of the sequence of solutions as the mesh parameter tends to zero. We have chosen the approach of $A$-proper mappings. For the definition we quote the references $[9,18]$.

THEOREM 8. The nonlinear operator $A(\cdot): L^{q}(\Gamma) \rightarrow L^{q}(\Gamma)$ is A-proper with respect to the projectionally complete scheme $\left\{P_{h}, S_{N}^{d}(\Theta)\right\}$.

Proof. The first defining property is that from every bounded sequence $\left\{u_{h_{j}}\right\}, u_{h_{j}} \in S_{N_{j}}^{d}\left(\Theta_{j}\right)$, for which $P_{h_{j}} A\left(u_{h_{j}}\right) \rightarrow f$ as $h_{j} \rightarrow 0$, we can subtract a subsequence $\left\{u_{h_{j(k)}}\right\}$ with the property: There exists a function $u$ 
such that

$$
\left\|u_{h_{j(k)}}-u\right\|_{L^{q}(\Gamma)} \rightarrow 0, \quad k \rightarrow \infty,
$$

and $A(u)=f$. Since $L^{q}(\Gamma)$ is reflexive, every bounded sequence has a weakly convergent subsequence. Let $\left\{u_{h_{j(k)}}\right\}$ be the subsequence with $u$ as the weak limit. By Theorem 1 , the operators $V$ and $K^{*}$ are compact from $L^{q}(\Gamma)$ onto $L^{p}(\Gamma)$ and $L^{q}(\Gamma)$ to $L^{q}(\Gamma)$, respectively. Via the basic properties of the linear compact operators we have

$$
\begin{gathered}
\left\|K^{*}\left(u_{h_{j(k)}}-u\right)\right\|_{L^{q}(\Gamma)} \rightarrow 0 \\
\left\|V\left(u_{h_{j(k)}}-u\right)\right\|_{L^{p}(\Gamma)} \rightarrow 0
\end{gathered}
$$

By the definition of the integral operator we have the identity

$$
u_{h_{j(k)}}=2\left(P_{h_{j(k)}} A\left(u_{h_{j(k)}}\right)-f\right)+2 f+2 P_{h_{j(k)}} K^{*} u_{h_{j(k)}}-2 P_{h_{j(k)}} G\left(V u_{h_{j(k)}}\right) \text {. }
$$

Therefore by the triangle inequality we get

$$
\begin{aligned}
& \left\|u_{h_{j(k)}}-2 f-2 K u+2 G(V u)\right\|_{L^{q}(\Gamma)} \\
& \quad \leq 2\left\|P_{h_{j(k)}} A\left(u_{h_{j(k)}}\right)-f\right\|_{L^{q}(\Gamma)}+2\left\|P_{h_{j(k)}} K^{*} u_{h_{j(k)}}-K^{*} u\right\|_{L^{q}(\Gamma)} \\
& \quad+2\left\|P_{h_{j(k)}} G\left(V u_{h_{j(k)}}\right)-G(V u)\right\|_{L^{q}(\Gamma)} .
\end{aligned}
$$

The right-hand side tends to zero by the assumption and (42). Thus the uniqueness of the weak limit in reflexive Banach spaces implies

$$
\frac{1}{2} u=f+K^{*} u-G(V u)
$$

and by (44) $u_{h_{j(k)}} \rightarrow u$ strongly in $L^{q}$-norm. Thus we have proved the first part of the statement. To prove the second part of the statement we have to show that $P_{h} D A(u)$ is continuous. But this is clear, since by the mapping properties of the integral operator $A(\cdot)$ and the $L^{2}$-projection $P_{h_{j}}: L^{q}(\Gamma) \rightarrow$ $L^{q}(\Gamma)$ are continuous.

The relevance of Theorem 8 is justified as follows: if one can prove the existence of bounded sequence of Galerkin solutions, then the $A$-properness will yield the convergence of the method.

Thus the problem is now to prove the existence of the Galerkin solutions. This we proceed to do by linearising the equations. The linearised operator has some rather nice properties. We have already seen that $A: L^{q}(\Gamma) \rightarrow$ $L^{q}(\Gamma)$ is continuously Fréchet differentiable. The Fréchet derivative acts on functions as

$$
D A(u) v=\frac{1}{2} u-K^{*} u+D G(V u) V v .
$$

But the derivative has even stronger properties that are crucial if we are to prove the unique solvability of the Galerkin equations. 
THEOREM 9. The bounded linear operator

$$
D A(u): L^{q}(\Gamma) \rightarrow L^{q}(\Gamma)
$$

is a Fredholm operator with index zero: $\operatorname{ind}(D A(u))=0$. Furthermore, it is also injective.

Proof. The first part of the statement is obvious from the general theory of Fredholm operators [23, Chapters IV and V]. Namely, the bounded linear operators $K^{*}$ and $D G(V u) V$ are compact by Theorem 1. Therefore $D A(u)$ is a Fredholm operator with index zero.

To prove the injectivity we shall show that $D A(u): L^{q}(\Gamma) \rightarrow L^{q}(\Gamma)$ is strictly $V$-monotone. As in Theorem 5 we use the semicoercivity of the linear operator $V\left(\frac{1}{2} I-K^{*}\right)$. There we noticed that for every $w \in L^{q}(\Gamma)$ we can find a harmonic function $\Psi \in W^{1,2}(\Omega)$ such that

$$
\left(V\left(\frac{1}{2} I-K^{*}\right) w, w\right)_{L^{2}(\Gamma)} \geq\|\nabla \Psi\|_{L^{2}(\Omega)}^{2} .
$$

The assumption A4 implies that $\frac{\partial}{\partial u} g(x, u)>0$ for almost all $x \in \Gamma$. Thus we get

$$
(D A(u) w, V w)_{L^{2}(\Gamma)}=\|\Psi\|_{L^{2}(\Omega)}^{2}+\int_{\Gamma} \frac{\partial}{\partial u} g(x, V u(x))|V w(x)|^{2} d s_{x}>0
$$

for every $w \neq 0$, which proves our statement.

Now we are in a position to prove the main theorem of this section.

THEOREM 10. For given $f \in L^{q}(\Gamma)$ there exists $h_{0}>0$ such that for every $0<h \leq h_{0}$ the Galerkin equations

$$
P_{h} A\left(u_{h}\right)=P_{h} f
$$

admit a unique solution, and $u_{h}$ converges towards the unique solution of the equation $A(u)=f$.

Proof. The statement is actually now a consequence of the general theory of $A$-proper mappings [9, Theorem 21.3, pp. 263-264] or [18, Theorem 4.3G]. But for the sake of convenience we recall the basic idea of the proof here. It relies strongly on the degree argument of the nonlinear Fredholm operators. By Theorem 8 there exists a constant $c=c(u)>0$ depending only on $u \in L^{q}(\Gamma)$ such that

$$
\|D A(u) v\|_{L^{q}(\Gamma)} \geq c(u)\|v\|_{L^{q}(\Gamma)}
$$

for all $v \in L^{q}(\Gamma)$ 
Next we shall estimate the operator norms of $\left(I-P_{h}\right) D G(V u) V$ and $\left(I-P_{h}\right) K^{*}$ in $L^{q}(\Gamma)$. Using the results for the approximation of compact operators [15, Section 8.5, p. 186] we obtain

$$
\begin{aligned}
\left\|\left(I-P_{h}\right) K^{*}\right\|_{L^{q} \rightarrow L^{q}} & \leq \delta(h), \\
\left\|\left(I-P_{h}\right) D G(V u) V\right\|_{L^{q} \rightarrow L^{q}} & \leq \delta(u ; h),
\end{aligned}
$$

where $\delta(h) \rightarrow 0$ as $h \rightarrow 0$. From these estimates it follows that

$$
\left\|\left(P_{h} D A(u)-D A(u)\right) v\right\|_{L^{q}(\Gamma)} \leq \delta(h)+\delta(u ; h) \rightarrow 0 .
$$

Thus the approximation of $D A(u)$

$$
A_{h}^{\prime}(u)=\frac{1}{2}-P_{h} K^{*}+P_{h} D G(V u) V
$$

is also an isomorphism in $L^{q}(\Gamma)$ provided the mesh parameter is small enough. As a consequence $P_{h} D A(u): S_{N}^{d}(\Theta) \rightarrow S_{N}^{d}(\Theta)$ is an homeomorphism, because the restriction of $A_{h}^{\prime}(u)$ to $S_{N}^{d}(\Theta)$ is $P_{h} D A(u)$. Hence the Brouwer degree of this finite dimensional operator

$$
d\left(P_{h} D A(u), B_{h}(0, r), 0\right)= \pm 1
$$

for every $r>0$. Notice that we can choose $r>0$ such that on the boundary of the set

$$
B_{h}(u, r)=\left\{w \in S_{N}^{d}(\Theta) \mid\|u-w\|_{L^{q}(\Gamma)} \leq r\right\}
$$

there holds

$$
P_{h} A(w) \neq P_{h} f
$$

This choice is possible, since we know that $u$ is the isolated solution of (23). The hard part of the proof is to show that [12, Chapter 1]

$$
d\left(P_{h} A(\cdot), B_{h}(u, r), P_{h} f\right)=d\left(P_{h} D A(u), B_{h}(0, r), 0\right)= \pm 1 .
$$

Since the degree of the finite dimensional operator is nonzero, there exists $u_{h} \in S_{N}^{d}(\theta)$ such that

$$
P_{h} A\left(u_{h}\right)=P_{h} f,
$$

if $h$ is sufficiently small. Furthermore, the Galerkin solutions are bounded. The convergence of the method follows now by the $A$-properness of the integral operator and the uniqueness of the continuous solution.

Let us finally consider the uniqueness of the Galerkin solutions. Since $A(\cdot)$ is continuously Fréchet-differentiable we can find $\rho>0$ such that for every $u_{h} \in B(u, \rho)$ there holds

$$
\left\|P_{h} D A\left(u_{h}\right) \phi\right\|_{L^{q}(\Gamma)} \geq c(u)\|\phi\|_{L^{q}(\Gamma)}, \quad \phi \in S_{N}^{d}(\Theta) .
$$


Let then $u_{h}, v_{h} \in S_{N}^{d}(\Theta)$ be Galerkin solutions on the same mesh, i.e.

$$
P_{h}\left[A\left(u_{h}\right)-A\left(v_{h}\right)\right]=0 .
$$

We choose $h_{0}>0$ so small that for every $h<h_{0}$ it holds that

$$
u_{h}, v_{h} \in B(u, \rho) \text { and }\left\|u_{h}-v_{h}\right\|_{L^{q}(\Gamma)}<\varepsilon / 2 \text {. }
$$

Here $\varepsilon / 2$ can be made so small that the remainder

$$
R\left(u_{h} ; u_{h}-v_{h}\right)<c(u) / 2 .
$$

Combining (47) and (48) with estimate

$$
\begin{aligned}
\left\|P_{h}\left[A\left(u_{h}\right)-A\left(v_{h}\right)\right]\right\|_{L^{q}(\Gamma)} \geq & \left\|P_{h} D A\left(u_{h}\right)\left(u_{h}-v_{h}\right)\right\|_{L^{q}(\Gamma)} \\
& -R\left(u_{h} ; u_{h}-v_{h}\right)\left\|u_{h}-v_{h}\right\|_{L^{q}(\Gamma)}
\end{aligned}
$$

we finally get

$$
0 \geq \frac{c(u)}{2}\left\|u_{h}-v_{h}\right\|_{L^{q}(\Gamma)},
$$

which proves the uniqueness.

Up to now we have not said a word about the accuracy of the approximate solution obtained by the Galerkin method. Next we shall use the Fredholm property to derive the asymptotic error estimates in $L^{q}(\Gamma)$-norm (compare with Theorem 4.4 in [19]). We begin by proving

LEMMA 11. For the Galerkin solutions there holds the following asymptotic estimates

$$
\left\|P_{h} u-u_{h}\right\|_{L^{q}(\Gamma)} \leq C(u)\left\{\left\|K^{*}\left(u-P_{h} u\right)\right\|_{L^{q}(\Gamma)}+\left\|V\left(u-P_{h} u\right)\right\|_{L^{q}(\Gamma)}\right\} .
$$

Proof. In Theorem 9 we proved that there exists $h_{0}>0$ such that for all $0<h<h_{0}$ we have

$$
\left\|P_{h} D A(u) \phi\right\|_{L^{q}(\Gamma)} \geq c(u)\|\phi\|_{L^{q}(\Gamma)}, \quad \phi \in S_{N}^{d}(\Theta) .
$$

Here $u \in L^{q}(\Gamma)$ is the unique solution of the nonlinear integral equation (23) as above. On the other hand we can replace $u$ in (50) by its orthogonal projection $P_{h} u$, because the nonlinear operator is continuously differentiable. Setting in (50) $\phi=P_{h} u-u_{h}$ we get

$$
\left\|u_{h}-P_{h} u\right\|_{L^{q}(\Gamma)} \leq c(u)\left\|P_{h} D A\left(P_{h} u\right)\left(u_{h}-P_{h} u\right)\right\|_{L^{q}(\Gamma)} .
$$

By the definition of the Fréchet-derivative

$$
D A\left(P_{h} u\right)\left(u_{h}-P_{h} u\right)=A\left(u_{h}\right)-A\left(P_{h} u\right)+R\left(P_{h} u ; u_{h}-P_{h} u\right)\left\|u_{h}-P_{h} u\right\|_{L^{q}(\Gamma)} \text {. }
$$

Theorem 9 and the properties of the $L^{2}$-projection imply

$$
\left\|u_{h}-P_{h} u\right\|_{L^{q}(\Gamma)} \rightarrow 0, \quad \text { as } h \rightarrow 0
$$


yielding finally the convergence of the remainder term in (52):

$$
R\left(P_{h} u ; u_{h}-P_{h} u\right) \rightarrow 0, \quad h \rightarrow 0 .
$$

In particular, we can choose $h_{0}$ small enough to get

$$
R\left(P_{h} u ; u_{h}-P_{h} u\right) \leq \frac{1}{8 c(u)} .
$$

Using the estimates (51), (52) and (53) together with the identity

$$
\begin{aligned}
P_{h}\left(A(u)-A\left(P_{h} u\right)\right)= & P_{h}\left(A\left(u_{h}\right)-A\left(P_{h} u\right)\right) \\
& -K^{*}\left(u-P_{h} u\right)+P_{h}\left[G(V u)-G\left(V\left(P_{h} u\right)\right)\right]
\end{aligned}
$$

we achieve

$$
\frac{7}{8 c(u)}\left\|u_{h}-P_{h} u\right\|_{L^{q}(\Gamma)} \leq\left\|K^{*}\left(u-P_{h} u\right)\right\|_{L^{q}(\Gamma)}+\left\|G(V u)-G\left(V P_{h} u\right)\right\|_{L^{q}(\Gamma)} .
$$

Here we have used also the boundedness of the $L^{2}$-projection in $L^{q}$-norm (see the estimate (39) with $s=t=0$ ).

To complete the argument, we have to estimate the second term on the right-hand side in (54). To do this we utilise the assumptions made on the nonlinearity. First we introduce some helpful notation to make the following estimates clearer. We set $v=V u$ and $v_{h}=V P_{h} u$. Then by the assumption A5 for almost all $x \in \Gamma$

$$
\left|G(v)(x)-G\left(v_{h}\right)(x)\right| \leq\left|v(x)-v_{h}(x)\right|\left\{K_{1}+K_{2}\left(|v(x)|^{(p-2)}+\left|v_{h}(x)\right|^{(p-2)}\right)\right\}
$$

With this inequality and the Hölder inequality we get

$$
\int_{\Gamma}\left|G(v)(x)-G\left(v_{h}\right)(x)\right| d s_{x} \leq\left\{K_{1}+K_{2}\left(\|v\|_{L^{\infty}}^{p-2}+\left\|v_{h}\right\|_{L^{\infty}}^{p-2}\right)\right\}\left\|v-v_{h}\right\|_{L^{q}(\Gamma)} \text {. }
$$

The right-hand side is finite since $V: L^{q}(\Gamma) \rightarrow C(\Gamma)$ is bounded by Theorem 1 . Using this property on the right-hand side of (55) we get the final estimate

$$
\left\|G(V u)-G\left(V P_{h} u\right)\right\|_{L^{q}(\Gamma)} \leq K\left(\|u\|_{L^{q}(\Gamma)}\right)\left\|V\left(u-P_{h} u\right)\right\|_{L^{q}(\Gamma)} .
$$

The previous lemma may be applied to establish the asymptotic error estimates in various Sobolev norms.

THEOREM 12. Let $u \in L^{q}(\Gamma)$ be the unique solution of the nonlinear integral equation $A(u)=f$, and $u_{h} \in S_{N}^{d}(\theta)$ the Galerkin solution corresponding to the mesh parameter $h=1 / N$. Then there holds the asymptotic stability estimates

and

$$
\left\|u-u_{h}\right\|_{L^{q}(\Gamma)} \leq c\left(\|u\|_{L^{q}(\Gamma)}\right)\left\|u-P_{h} u\right\|_{L^{q}(\Gamma)}
$$

$$
\left\|u-u_{h}\right\|_{W^{-1, q}(\Gamma)} \leq c_{1}\left(\|u\|_{L^{q}(\Gamma)}\right)\left\|u-P_{h} u\right\|_{W^{-1, q}(\Gamma)}
$$


Proof. The first part of the statement is obvious. By the triangle inequality we obtain

$$
\left\|u-u_{h}\right\|_{L^{q}(\Gamma)} \leq\left\|u-P_{h} u\right\|_{L^{q}(\Gamma)}+\left\|u_{h}-P_{h} u\right\|_{L^{q}(\Gamma)} .
$$

For the second term on the right hand side we can use estimate obtained in Lemma 11. If we choose the mesh parameter $h<h_{0}$, we have

$$
\begin{aligned}
\left\|u-u_{h}\right\|_{L^{q}(\Gamma)} \leq & \left\|\left(I-P_{h}\right) u\right\|_{L^{q}(\Gamma)} \\
& +c\left(\|u\|_{L^{q}(\Gamma)}\right)\left\{\left\|K^{*}\left(u-P_{h} u\right)\right\|_{L^{q}(\Gamma)}+\left\|V\left(u-P_{h} u\right)\right\|_{L^{q}(\Gamma)}\right\} .
\end{aligned}
$$

Because the operators $V: L^{q}(\Gamma) \rightarrow L^{q}(\Gamma)$ and $K: L^{q}(\Gamma) \rightarrow L^{q}(\Gamma)$ are bounded (Theorem 1), we get

$$
\left\|u-u_{h}\right\|_{L^{q}(\Gamma)} \leq\left\{1+c\left(\|u\|_{L^{q}(\Gamma)}\right)\left(\left\|K^{*}\right\|+\|V\|\right)\right\}\left\|u-P_{h} u\right\|_{L^{q}(\Gamma)} .
$$

The estimate (57) follows by utilising the mapping properties of the operators $V$ and $K^{*}$. Since we assumed that the boundary curve $\Gamma$ is smooth, there holds: for every $w \in W^{-1, q}(\Gamma)$

$$
\begin{aligned}
\|V w\|_{L^{q}(\Gamma)} & \leq c\|w\|_{W^{-1, q}(\Gamma)}, \\
\left\|K^{*} w\right\|_{L^{q}(\Gamma)} & \leq c\|w\|_{W^{-1, q}(\Gamma)}
\end{aligned}
$$

With these estimates and Lemma 11 one easily deduces

$$
\left\|u_{h}-P_{h} u\right\|_{W^{-1, q}(\Gamma)} \leq c\left(\|u\|_{L^{q}(\Gamma)}\right)\left\{\left\|K^{*}\right\|+\|V\|\right\}\left\|u-P_{h} u\right\|_{W^{-1, q}(\Gamma)} .
$$

But this estimate already proves the inequality (57), because by the triangle inequality we have

$$
\left\|u-u_{h}\right\|_{W^{-1, q}(\Gamma)} \leq\left\|u-P_{h} u\right\|_{W^{-1, q}(\Gamma)}+\left\|u_{h}-P_{h} u\right\|_{W^{-1, q}(\Gamma)} .
$$

Theorem 12 yields immediately the asymptotic convergence rates provided we know the regularity of the solution $u$.

COROLlaRY 13. Let us assume that the solution $u \in W^{s, q}(\Gamma)$. Then there holds the error estimates

$$
\left\|u-u_{h}\right\|_{W^{t, q}(\Gamma)} \leq c\left(\|u\|_{L^{q}(\Gamma)}\right) h^{s-t}\|u\|_{W^{s, q}(\Gamma)},
$$

for every $-1 \leq t \leq 0 \leq s \leq d+1$.

Proof. By Theorem 12 the estimates follow simply by the mapping properties of spline functions.

Finally we want note that these theoretical results agree quite well with the numerical computations made in [21, Example 2]. There the piecewise constants are used to approximate the solution. The experimental order was 
exactly the theoretical order obtained here. The numerical computations were carried out with the $|u| u^{3}$-nonlinearity.

\section{Acknowledgement}

This research was performed while the author was visiting at the University of New South Wales (1989). The author is grateful to the Australian Research Council for providing a grant for the research, and to Professor I. Sloan for arranging working facilities.

\section{References}

[1] R. A. Adams, Sobolev spaces (Academic Press, New York, 1975).

[2] D. Arnold and W. L. Wendland, "On the asymptotic convergence of collocation methods", Math. Comp. 41 (1983) 349-381.

[3] I. Babuška and A. K. Aziz, "Survey lectures on the mathematical foundation of the finite element methods", The mathematical foundation of the finite element method with applications to partial differential equations (Academic Press, New York, 1972) 3-359.

[4] V. Barbu, Nonlinear semigroups and differential equations in Banach spaces (Noordhoff International Publishing, Leyden, 1978).

[5] H. Brézis, "Problemés unilatereaux", J. Math. Pures et Appliquées 51 (1972) 1-168.

[6] M. Costabel, "Starke elliptizität von Randintegraloperatoren erster Art", Habilitationsschrift Darmstadt 1984.

[7] M. Costabel and E. Stephan, "Boundary integral eqautions for mixed boundary value problem in polygonal domains and Galerkin approximation", Mathematical Models and Methods in Mechanics (Polish Scientific Publ. 1985).

[8] C. de Boor, "A bound on the $L^{\infty}$-norm of $L^{2}$-approximation by splines in terms of global mesh ratio", Math. Comp. 30 (1976) 765-771.

[9] K. Deimling, Nonlinear functional analysis (Springer-Verlag, Heidelberg, 1985).

[10] P. Eggermont and J. Saranen, “ $L^{p}$ estimates of boundary integral equations for some nonlinear boundary value problems" (to appear).

[11] J. Elschner and G. Schmidt, "On spline interpolation in periodic Sobolev spaces", P-Math-01/83 (Akad. Wiss. DDR, Inst. Math., Berlin 1983).

[12] S. Fučik, J. Nečas, J. Souček and V. Souček, Spectral analysis of nonlinear operators (Springer Verlag, Berlin-Heidelberg-New York, 1973).

[13] W. Hackbusch and Nowak, "On the fast matrix multiplication in the boundary element method by panel clustering", Numer. Math. 54 (1989) 463-491.

[14] M. Hamina, K. Ruotsalainen and J. Saranen, "Numerical approximation for the solution of a nonlinear boundary integral equation with the collocation method", J. Int. Eqs. and Appl. 4 (1992) 1-21.

[15] K. Jörgens, Linear integral operators (Teubner, Stuttgart, 1970).

[16] J. T. Oden, Qualitative methods in nonlinear mechanics (Prentice-Hall, New Jersey, 1986).

[17] D. Pascali and S. Sburlan, Nonlinear mappings of monotone type (Siijthoff and Noordhoff International Publ., Bucarest, 1978). 
[18] W. V. Petryshyn, "On the approximation-solvability of equations involving $A$-proper and pseudo A-proper mappings", Bull. Amer. Math. Soc. 81 (1975) 223-312.

[19] K. Ruotsalainen, "On the the boundary element method for a mixed non-linear boundary value problem", Applicaple Analysis (to appear).

[20] K. Ruotsalainen and J. Saranen, "On the collocation method for a nonlinear boundary integral equation", J. Comp. Appl. Math. 28 (1989) 339-348.

[21] K. Ruotsalainen and W. L. Wendland, "On the boundary element method for some nonlinear boundary value problems", Numer. Math. 53 (1988) 299-314.

[22] J. Saranen, "Projection methods for a class of Hammerstein equations", SIAM J. Numer. Anal. (to appear).

[23] M. Schechter, Principles of functional analysis (Academic Press, New York, 1971).

[24] L. Schumaker, Spline functions: Basic theory (John Wiley, New York, 1981).

[25] M. M. Vainberg, Variational methods for the study of nonlinear operators (Holden-Day, San Francisco, 1964).

[26] G. Verchota, "Layer potentials and regularity for the Dirichlet problem for Laplace's equation in Lipschitz domains", J. Funct. Anal. 59 (1984) 572-611. 